\title{
EL PROTOCOLO “NETWORK" APLICADO AL ESTUDIO DE LOS DESTINOS TURÍSTICOS: EL CASO DE GIJÓN (ESPAÑA)
}

\author{
Francisco Javier de la Ballina Ballina \\ Universidad de Oviedo \\ fballina@uniovi.es \\ ORCID iD: https://orcid.org/0000-0001-7553-1066
}

Recibido: 10-12-2018 Aceptado: 07-05-2020

Cómo citar este artículo/Citation: Ballina Ballina, Francisco Javier de la (2020). El protocolo "network" aplicado al estudio de los destinos turísticos: el caso de Gijón (España). Estudios Geográficos, 81 (288), e041. https://doi.org/10.3989/estgeogr.202056.056

\begin{abstract}
RESUMEN: La definición del concepto de destino es un asunto muy complejo, dada la necesidad de consenso entre distintos tipos de académicos y de profesionales, pero muy necesaria para poder establecer un marco adecuado de conocimiento orientado a la planificación y a la gestión del turismo. La mayoría de las nuevas propuestas giran en torno a la perspectiva de Red (Network), con intra e interrelaciones entre agentes, atracciones y servicios turísticos, e incluso destinos. El factor experiencia de "segunda generación", apoyado en el fenómeno de la co-creación, es actualmente considerado el leit motiv para el turista. De acuerdo a tal concepto son los turistas quienes, con las elecciones de recursos y servicios, y con sus movimientos en los territorios, definen realmente un destino como tal, y en sus diferentes facetas. Este trabajo recurre al uso del conocido juego del SmartMax para ofrecer un protocolo empírico de conocimiento, cuantitativo y cualitativo, de un destino turístico. Apoyándose en datos sobre la movilidad de los turistas, y mediante técnicas de análisis multivariable, se consigue identificar los diferentes perfiles de un espacio geográfico como destino turístico, así como el peso relativo de cada uno de ellos: destino único, sede central, destino de circuito y destino de excursionismo. De esta forma se construye una metodología útil para el conocimiento empírico de los destinos turísticos, de forma general, e incluso para su aplicación en otros ámbitos del marketing geográfico, en particular.
\end{abstract}

PALABRAS CLAVE: Destinos turísticos; Co-creación; Flujos turísticos; Estadísticas turísticas; Mapas turísticos; Productos turísticos.

\section{THE “NETWORK” PROTOCOL APPLIED TO THE STUDY OF TOURIST DESTINATIONS: THE CASE OF GIJON (SPAIN)}

ABSTRACT: The definition of the concept of destination is a very complicated matter, given the need for consensus between different types of academics and professionals. However, it is essential to establish an adequate framework of knowledge for planning and managing tourism. Most of the new proposals revolve around the Network perspective, with intra and interrelationships between agents, attractions and tourist services, and even destinations. The "second generation" experience factor, supported by the phenomenon of co-creation, is currently considered the leitmotif for tourists. According to this concept, it is the tourists who, with the choices of resources and services, and with their movements in the territories, really define a destination as such, and in its different facets. This work uses the well-known SmartMax game to offer an empirical protocol of knowledge, quantitative and qualitative, of a tourist destination. Relying on data on the mobility of tourists, and using multivariable analysis techniques, it is possible to identify the different profiles of geographic space as a tourist destination, as well as the relative weight of each one: single destination, headquarters, a destination of circuit and hiking destination. In this way, a useful methodology is built for the empirical knowledge of tourist destinations, in general, and even for its application in other areas of geographic marketing, in particular.

KEY WORDS: Tourism destinations; Co-creation; Touristic Flows; Tourist Statistics; Tourism maps; Tourism Products.

Copyright: ( 2020 CSIC. Este es un artículo de acceso abierto distribuido bajo los términos de la licencia de uso y distribución Creative Commons Reconocimiento 4.0 Internacional (CC BY 4.0). 


\section{INTRODUCCIÓN}

El concepto de destino turístico se mantiene en una indefinición que genera serios problemas para facilitar su planificación y gestión. Sorprende que, siendo tan trascendente para la actividad turística, permanezca en esta imprecisión conceptual (Moragues, 2006). Recientemente Pearce (2014) ha criticado la excesiva fragmentación de la investigación sobre el destino y la carencia de paradigma que sustente su definición.

La definición oficial de la OMT (2008) es criticada desde todas las posiciones: "el destino principal de un viaje turístico se define como el lugar visitado que es fundamental para la decisión de realizar el viaje" (Glosario, párr. 2.31). Para los economistas por carecer de un enfoque de producción, para los biólogos, sociólogos y geógrafos por no prestar atención a los efectos sobre todo "lo local".

A este pulso académico, casi tradicional, se está añadiendo la radical transformación, que sufre tanto la oferta como la demanda, de los servicios turísticos en los últimos años. Efectivamente, se asiste a una rápida transformación del modelo "postfordista" originada por las tendencias tecnológicas, el auge de la calidad y de la sostenibilidad, y los cambios motivacionales y experienciales.

El uso, y consumo, del destino turístico pasa a ser, progresivamente, más diverso y flexible, en el que juega un rol más determinante el turista, lo que ha llevado a proponer el factor de la "co-creación" en el turismo (Gretzel et al. 2015).

La concepción de un destino único, cerrado por unas fronteras, más o menos administrativas, ya no es adecuada para unos turistas que crean sus propios circuitos y rutas, incluso entre puntos geográficamente lejanos. Nuevos conceptos de gestión, como el de destino en sede central, destino en circuito $y$, de forma notable, los excursionismos fronterizos desbordan a los gestores del destino, poniendo en tela de juicio la definición de un destino turístico.

La academia necesita disponer de nuevas propuestas empíricas para desarrollar el paradigma conceptual del destino turístico. En la actualidad, existe cierto consenso en caminar por la senda del modelo en red (Network Destination). Con tal objetivo, este trabajo recurre al didáctico juego del Smart-Max (arquitectura con imanes) como protocolo aplicado del enfoque de destino en red, que, además, pueda ayudar a comprender las necesidades de planificación y gestión de cada multi-destino. $Y$ todo ello con una perspectiva de cooperación intraterritorial y extraterritorial.

Para ello, el trabajo se estructura en tres principales apartados. En primer lugar, se debate sobre las definiciones de destino turístico presentadas por las diferentes escuelas académicas, a lo largo del tiempo. Luego, se presenta el concepto de co-creación turística como el paradigma más interesante para la construcción de las experiencias turísticas. Finalmente, se desarrollan los principios del protocolo SmartMax, la metodología para su desarrollo y los resultados obtenidos de su aplicación práctica sobre el caso concreto de Gijón (Asturias, España).

\section{LAS ESCUELAS DEL DESTINO TURÍSTICO}

Tradicionalmente la literatura ha seguido dos ópticas de estudio del destino bien distintas: las escuelas económica y territorial. Para la escuela territorial, liderada por los geógrafos, el turismo es una actividad espacial (Alberca, 2016) siendo el destino: el espacio - país, región o ciudad-al que llega el viajero (Martínez y Rojo, 2013). Vera (2011) amplía la cuestión de la territorialidad añadiendo la dimensión temporal. De esta forma, la distancia y los desplazamientos turísticos son los fundamentos para comprender el destino turístico. Las principales preocupaciones de esta escuela académica han sido las cargas sobre el destino, como un todo, y sobre cada uno de sus recursos turísticos, en concreto (Vera y Baños, 1997).

Por su parte, los muchos autores de la escuela económica entienden el destino turístico como el contenedor del producto final que consume el turista, bien sea directamente, como un todo, o bien sea agregando distintos consumos dentro de un espacio territorial. Sin duda, una de las aportaciones más importantes para la gestión de los destinos de esta escuela es el desarrollo conceptual del factor "imagen del destino". De esta manera, los turistas generan expectativas en relación a la información que reciben y perciben del destino (Cooper et al., 2005, Kladou y Kehagias, 2014) que luego se contrastarán en los recursos y servicios turísticos finalmente utilizados.

Un primer enfoque mixto lo plantea Boullón (2004), quien destaca la elevada confusión existente entre los conceptos de destino y de espacio turístico. El autor plantea que el espacio es diverso y entrecortado, pues solo debería abarcar aquella parte que efectivamente utiliza el turista. Esta aportación sintoniza 
con el concepto de "zona geoturística" de Vera (2011), que incluye los elementos de: imagen, promoción y comercialización del destino. Si bien, dando una importancia crítica al estudio del área de influencia espacial de cada atractivo turístico.

En los últimos años, han abundado en la literatura sobre destinos turísticos las propuestas integradoras al estilo de Boullón (2004). Este tipo de enfoques se han venido apoyando en tres conceptos principales: Sistema, Constructo y de Red (Pierce, 2012) (ver figura 1).

La visión del destino como un sistema fue planteada inicialmente por Framke (2002), con un gran apoyo y desarrollo posterior. Abundante literatura apoya la definición del destino turístico considerando, no sólo la suma de sus elementos individuales, sino, y, sobre todo, las interacciones que se producen entre éstos (internos) y con otros (externos) (Alberca, 2016; Baggio y Sainaghi; 2014; Beritelli y Bieger; 2014). Esta Escuela del Sistema propone dos facetas del destino fuertemente interrelacionadas, al modo de la cara y cruz de una moneda, que son:

1. El subsistema de demanda que se centra en las interrelaciones entre los proveedores de bienes y servicios y los turistas (Manente y Cerato, 2000). La idea es que los turistas perciben el producto/servicio turístico como un "unicum" (Bonetti et al., 2006).

2. El subsistema espacial que se ocupa de los enlaces entre los distintos lugares turísticos: el elemento clave serán los flujos turísticos (mayores o menores, principales o secundarios, interregionales o intrarregionales, intercontinentales o intracontinentales), que, además, estarían cambiando permanentemente a lo largo del tiempo (Vera, 2011).

El segundo enfoque integrado se construye sobre la definición del "Constructo". Fue propuesto por diversos autores franceses, bajo el liderazgo de Violier (2009), si bien ha tenido una influencia limitada. Bajo tal propuesta se plantea que: un mismo destino turístico es percibido e interpretado de forma distinta por cada turista, porque lo hace en función de sus principios sociales y culturales, que son bien distintos y, además, subjetivos (Saraniemi y Kylanen, 2011).

El modelo integrado de destino más desarrollado actualmente, y calificado por diversos autores como el más potente (Casanueva et al., 2014; Pearce, 2012,

FIGURA 1

EVOLUCIÓN DE LAS ESCUELAS DEL DESTINO TURÍSTICO

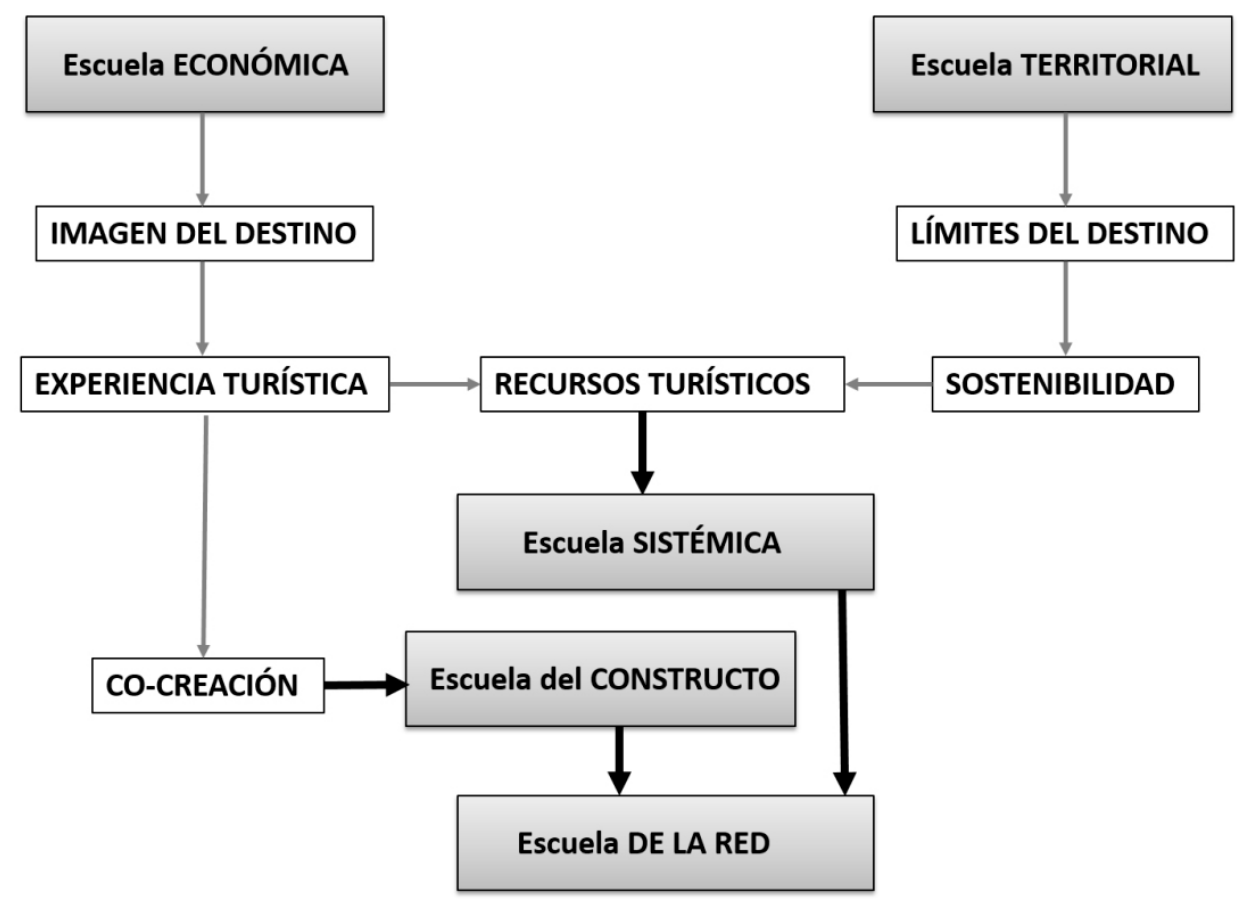

Fuente. Elaboración propia. 
2014), es el enfoque de "Destino en Red" (Network Destination). El elemento clave es la combinación de recursos turísticos personalizada por cada turista, y cambiante en el tiempo. De esta forma cobran valor explicativo lo movimientos, no solo dentro de un mismo territorio, sino, también, en sus interrelaciones con otros (D’Agata et al. 2013; Sang-Hyun et al. 2013).

El enfoque del Destino en Red está siendo utilizado, de forma creciente, gracias al denominado "Social Network Analysis" (SNA) (Denicolai et al., 2010; Merilainen y Lemmetyinen, 2011; Merinero y Pulido, 2009). El SNA opera con dos componentes principales: los nudos y los conectores, con sus distintas combinaciones construye varios indicadores definitorios de un destino turístico: densidad, centralidad, cohesión, tamaño, protuberancia, nivel de formalidad, grado de estabilidad, grado de flexibilidad, y fuerza de unión.

\section{EL PAPEL DE LA EXPERIENCIA Y LA CO-CREACIÓN EN EL DESTINO}

El rol de la demanda como factor de conceptualización de los destinos turísticos ha tenido un desarrollo importante con la implementación del paradigma de la experiencia turística. En este sentido, todo viajero vive una experiencia, o conjunto de experiencias, cuando viaja por turismo, y no sólo en su relación directa con lo que ofrece el destino, sino también a partir de las motivaciones para realizar el viaje, y a su posición activa dentro del mismo (Elands y Lengkeek, 2012). Las experiencias pueden "emocionar" a las personas, son intangibles e inmateriales, pero son quienes aportan valor al destino turístico, puesto que son memorables (Mehmetoglu y Engen, 2011).

De este paradigma surge, en primer lugar, el término "Prosumidor": un turista que consume y produce información de forma simultánea (Ritzer et al., 2012). Se está ante el "e-tourism" de Buhalis (2003), con turistas que utilizan las TICs para el antes (reservas) y/o para el después (opiniones). Actualmente, la cuestión tecnológica cobra mayor protagonismo durante el viaje, se funde como una parte más del mismo (Li et al., 2017). Esta adición TICs genera el salto hacia el término "AdProsumidor", caracterizado por ser un turista: conectado permanentemente, fundamentado en las recomendaciones, que se sabe influyente, conocedor de mucha información del destino, y que, además, tienda a comportamientos turísticos alternativos (González, 2017). Esto es, un turista de co-creador, que añade valor a su experiencia turística, con una fuerte interacción con los atractivos, agentes turísticos, y con los demás turistas (Buonincontri et al., 2017; Chathoth et al., 2016).

Ciertamente, el concepto de la co-creación lleva tiempo en la literatura académica: Kambil et al. (1999) fueron los primeros en plantearlo; Gronroos (2008) lo propuso para dar fundamento al marketing de servicios; y más recientemente, Kotler et al. (2017) lo incluyen como uno de los cuatro pilares (Ps) del nuevo marketing tecnológico. No existe una definición única de co-creación: el trabajo de García (2018) presenta hasta 52 definiciones distintas. Pero sí existe un cierto consenso, en el caso del turismo, en que "en el proceso de co-creación de valor los consumidores no aportan únicamente opiniones, deseos y necesidades, sino que contribuyen con su creatividad y habilidades en la resolución de problemas y el desarrollo de nuevos productos" (Füller, $2010 ; 120)$. Esto es, el turista es un recurso que debe ser integrado en un espacio turístico conectado (Ind et al., 2013).

Lo importante es entender la evolución del papel de la co-creación en el turismo: (1) de colaboración con el turista, de tal manera que todos los agentes turísticos deben centrar sus esfuerzos en la atención y el servicio a sus demás (la "lógica del servicio") (Vargo et al., 2008); (2) de interacción con el turista, muy importante por la gran cantidad de puntos de contacto entre los turistas y los agentes turísticos (Sorensen y Jensen, 2015); y (3) de creatividad, porque los turistas actúan como diseñadores de sus propias soluciones (Martini et al., 2014), consiguiendo experiencias más auténticas y flexibles (Richards, 2011).

Las tecnologías "mobile" tienen un impacto significativo en las actitudes de los turistas y en sus comportamientos, lo que afecta directamente a los destinos (Liu et al., 2014). Se trata de tecnologías que crean una piel "hiperconectada" en el cuerpo de los turistas (Rabari y Storper, 2015) facilitando la creación de sus propias experiencias turísticas (Gretzel et al, 2016). Este importante salto tecnológico general el concepto de co-creación de segunda generación a partir de la nueva experiencia "phygital": "the phygital experience consists in hybridizing the physical and digital components at the same time and in the same place" (Belghiti et al., 2017, 61). Sobre el que se construyen los actuales modelos de gestión del destino denominados Destinos Inteligentes (Smart Tourism Destinations). 


\section{OBJETIVOS E HIPÓTESIS}

El presente trabajo desarrolla un protocolo práctico para estudiar los destinos, dentro de lo que podría denominarse la Escuela de Red (Network Destination). Esta propuesta utiliza la mecánica del juego de imanes SmartMax. No obstante, frente a las metodologías SNA (que exigen variables únicamente métricas), este caso opera combinando procedimientos de análisis multivariable nominales (Sí/No), en coherencia con el carácter multidimensional del concepto de destino turístico. El objetivo es disponer de un protocolo práctico, sistematizado, sencillo de aplicar, y de validez general a cualquier tipo y caso de destino. Si ello se consigue, se podrá mejorar la comprensión del destino y su desarrollo dentro del interesante Enfoque de Red. Para ello se concretan las siguientes hipótesis:

$\mathrm{H} 1$ : El comportamiento co-creativo -de la experiencia- de los turistas determina que los flujos (o movimientos) constituyan el factor principal para la multi-definición del destino turístico.

H1.1: Los flujos (o movimientos) de los turistas hacia, y entre, las atracciones turísticas internas y externas amplían la dimensión geográfica del destino.

H1.2: El destino turístico es ampliado geográficamente cuando atrae hacia sus atracciones un número elevado de turistas que pernoctan en áreas colindantes, creando una red de satélites.

H1.3: Los flujos (o movimientos) de los turistas hacia, y entre, los servicios turísticos de pernoctación amplían la dimensión geográfica del destino.

$\mathrm{H} 2$ : El comportamiento co-creativo -de la experiencia- de los turistas se consolida con el de otros similares generando una jerarquía de rutas de experiencias -atracciones y servicios- dentro el destino turístico.

\section{Protocolo SMARTMAX De destino tURístico}

La conjunción del paradigma de la co-creación con los principios de la Escuela de Red determina la asunción de que cada turista genera su propio destino turístico: es el agente turístico principal que utiliza las diversas piezas, independientes y/o interrelacionadas, que se le ofrecen dentro de un destino. Bajo este planteamiento se propone el recurso al conocido juego del SmartMax: cada turista utilizará las piezas disponibles que le interesen para su desarrollar la experiencia, dando así forma al destino turístico. Las piezas del SmartMax son de tres tipos: los nodos los conectores y la plantilla (ver figura 2).

\section{FIGURA 2}

PIEZAS DEL JUEGO SMART MAX PARA EL DESTINO TURÍSTICO
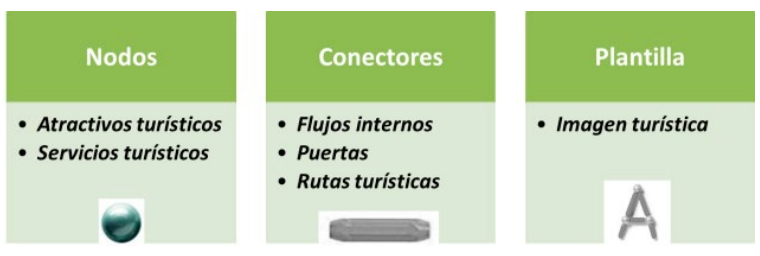

Fuente. Elaboración propia.

Los atractivos turísticos son el elemento primario en el desarrollo del concepto del destino (Navarro, 2015) pues la experiencia turística se construye a partir del uso y aprovechamiento de los mismos. De forma similar, la imagen del destino está fuertemente influenciada por la cantidad y calidad de sus atractivos. Es por ello que, en el juego SmartMax, los atractivos se configuran como los nodos/imanes -Lohmann y Pearce, 2010, comenzaron a plantear la denominación de nodos- que permiten crear y dar forma al destino turístico. Los servicios turísticos sustentan y mejoran las experiencias turísticas relacionadas con las atracciones, por lo tanto, toman, también, la forma de nodos-imanes, que pueden estar integrados, en algunos casos, dentro de las atracciones $y$, en otros, más distantes de las mismas.

Los conectores, por su parte, representan los movimientos físicos -Keum, 2010, es pionero en defender el papel clave de los flujos para el destino- que realiza el turista dentro de "su" concepto de destino, ya sean para llegar o marchar del mismo, para comunicar unos nodos de atracciones con otros y/o con nodos de servicios. El estudio y la gestión de los conectores (Marrocu y Paci, 2013; Morley et al., 2014; Yang, 2012) resultan críticos para entender el concepto multi-dimensional de destino, sea por la diversidad de experiencias turísticas -tipos de productos-, sea por las cargas que suponen los movimientos de los turistas en el territorio -masificación, sostenibilidad-. En consecuencia, el protocolo SmartMax centra la máxima atención en los conectores, según dos cri- 
terios principales que, cruzados, generan cuatro tipologías:

1. Flujos internos cortos: se producen dentro de un espacio geo turístico para vivir una experiencia que se encuentra en su interior. Están directamente relacionados con el tradicional concepto de destino único: los turistas llegan en grandes sistemas de transporte para quedarse durante toda su estancia en un mismo lugar, o al menos, en un radio geográfico limitado del mismo.

2. Flujos internos largos: conectan nodos de experiencia que requieren desplazamientos a atracciones propias de otro espacio geo turístico próximo, normalmente colindante, se trata de movimientos característicos del turista del caso destino de sede central.

3. Flujos externos cortos: son los utilizados por los excursionistas, que visitan determinadas atracciones del destino sin pernoctar en el mismo, obviamente, desde la perspectiva del destino que los recibe, son justamente los inversos al tipo anterior.

4. Flujos externos largos: supone la conexión larga que une el origen del turista y el destino, es el caso más frecuente, ahora bien, si el origen es otro espacio geo-turístico en el que ha pernoctado aparecería la dimensión de circuito turístico.

Finalmente, la literatura académica ha destacado el rol de la "imagen" del destino. Es el componente psicosociológico -el constructo- del destino para el turista, esto es, el sentimiento personal que el turista tiene de cada lugar. La imagen del destino determina la elección o no del mismo, de los nodos de atracciones, de los nodos de servicios, de los flujos previstos y de los, finalmente, realizados. En consecuencia, la imagen sirve de guía o "modelo" para realizar su propio montaje del destino, por eso el protocolo SmartMax utilizará el término de "plantilla".

La figura 3 representa un ejemplo del juego SmartMax con sus diferentes piezas.

\section{Metodología de APLicación del PROTOCOlO SMARTMAX}

Tal como ha indicado Boullón (2004), la única forma de definir un destino turístico es a través del empirismo. Pearce (2014) señala, también, cómo el estu-

APLICACIÓN DEL MODELO SMART MAX PARA UN DESTINO TURÍSTICO

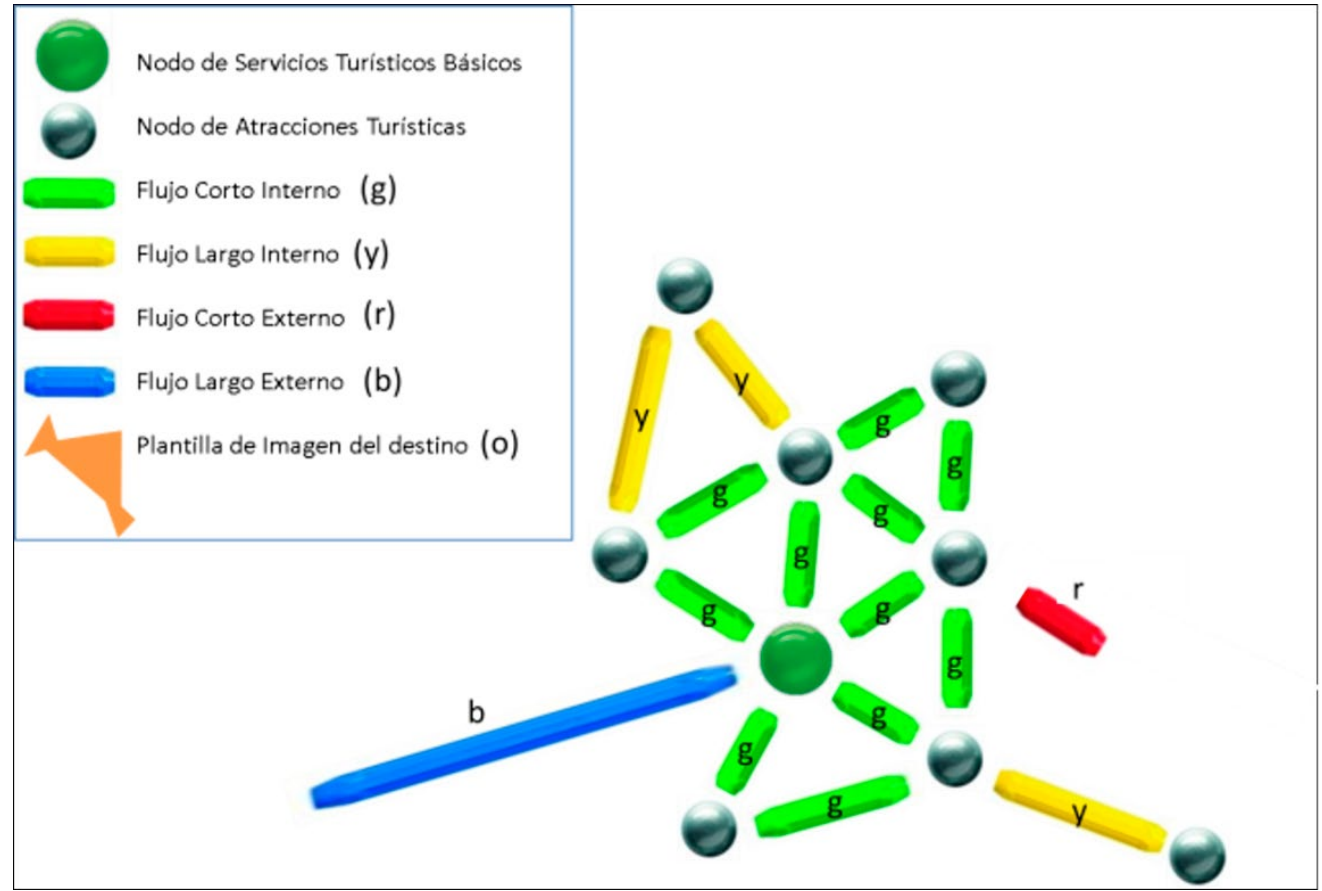

Fuente. Elaboración propia. 
dio empírico del destino se ve favorecido con el recurso a un Modelo de Red porque: (a) es superior en precisión matemática; (b) permite reflejar las particularidades locales de cada destino; y (c) dota al destino de una mayor agilidad y dinamismo conceptual.

La aplicación concreta del protocolo SmartMax fue realizada para la ciudad de Gijón -la mayor de Asturias y la segunda en plazas hoteleras del norte de España-. La elección ha estado motivada por su mix de turismo vacacional, cultural y de negocios, porque ello facilita la existencia de experiencias turísticas bien distintas y con diferente estacionalidad. Los datos utilizados se han obtenido del Sistema de Información Turística de Asturias (SITA), a través de sus encuestas en destino, durante los años 2012 a 2016. Las variables controladas son de dos tipos: el lugar o lugares de pernoctación, y las atracciones visitadas en su viaje (ver tablas 1 y 2 ).

El desarrollo metodológico se produce en un total de seis etapas, que permiten ir discriminando cada una de las variables de movilidad y/o pernoctación (ver figura 4). Como quiera que tales variables son de tipo categórico (Si/No pernocta; Si/No visita atractivo turístico) se recurre al uso de técnicas multivariables no-métricas, en concreto: el Análisis Discriminante Simple para estudiar el caso de las pernoctas externas, y la Escala Multidimensional No-Métrica para estudiar las interrelaciones de las atracciones turísticas (ver figura 5), de manera que:
TABLA 1

FICHA TÉCNICA DEL ESTUDIO EMPÍRICO

\begin{tabular}{|l|c|}
\hline Población & Turistas que visitan Gijón \\
\hline Muestra & 16.455 turistas \\
\hline $\begin{array}{l}\text { Procedimiento } \\
\text { de muestreo }\end{array}$ & $\begin{array}{c}\text { Cuotas por establecimiento y rutas } \\
\text { aleatorias por atracciones turísticas }\end{array}$ \\
\hline Tipo de encuesta & Personal \\
\hline $\begin{array}{l}\text { Nivel de } \\
\text { Confianza }\end{array}$ & Al 95\%, P $=0=0,5$ \\
\hline $\begin{array}{l}\text { Tamaño del } \\
\text { Error }\end{array}$ & Región de Asturias \\
\hline Ámbito muestral & Anual, 2012, 2013, 2014, 2015 y 2016 \\
\hline $\begin{array}{l}\text { Momento } \\
\text { muestral }\end{array}$ & Sistema de Información Turística de Asturias \\
\hline SITA
\end{tabular}

Fuente: Elaboración propia.

- Para las hipótesis del tipo 1 , sobre flujos de atracciones y recursos, se han efectuado mediante sendos Análisis Discriminantes Simples (ADS) del IBM SPSS, esto es: de destino único versus de sede central; de destino en circuito versus no en circuito; y de excursionista versus no excursionista.

- Para la hipótesis 2, sobre la agrupación de las atracciones turísticas, por sus correlaciones para los mismos turistas, se utiliza la aplicación de una Escala Multidimensional No-Métrica, me-

TABLA 2

VARIABLES UTILIZADAS EN EL ESTUDIO EMPÍRICO

\begin{tabular}{|c|c|c|c|c|}
\hline \multicolumn{3}{|c|}{ Alojamiento actual } & \multicolumn{2}{|c|}{ Municipio } \\
\hline \multicolumn{3}{|c|}{ Alojamiento anterior o posterior } & \multicolumn{2}{|c|}{ Municipio o Región o País } \\
\hline $\begin{array}{l}\text { Atracciones turísticas } \\
\text { visitadas de Asturias }\end{array}$ & $\begin{array}{c}\text { Narcea } \\
\text { Muniellos } \\
\text { Cudillero } \\
\text { Tapia } \\
\text { Luarca } \\
\text { Castropol-Eo } \\
\text { Navia } \\
\text { Coaña } \\
\text { Taramundi } \\
\text { Teixois }\end{array}$ & $\begin{array}{c}\text { Navelgas } \\
\text { Oscos } \\
\text { Avilés } \\
\text { Cabo Peñas } \\
\text { Gijón } \\
\text { Pravia-Salas } \\
\text { Oviedo } \\
\text { Senda del Oso } \\
\text { Somiedo } \\
\text { Mumi }\end{array}$ & $\begin{array}{c}\text { Lena } \\
\text { Aller } \\
\text { Caso-Redes } \\
\text { Nava } \\
\text { Villaviciosa } \\
\text { Lastres } \\
\text { Ribadesella } \\
\text { Llanes } \\
\text { Mirador Fitu } \\
\text { Cangas Onís }\end{array}$ & $\begin{array}{c}\text { Covadonga } \\
\text { Los Lagos } \\
\text { Rutas/Picos Europa } \\
\text { Localidades Picos de Europa } \\
\text { Otros Centro } \\
\text { Otros Oriente } \\
\text { Otros Occidente }\end{array}$ \\
\hline $\begin{array}{l}\text { Atracciones turísticas } \\
\text { visitadas de Gijón }\end{array}$ & $\begin{array}{l}\text { La Laboral } \\
\text { Jardín Botánico } \\
\text { Pueblo Asturias } \\
\text { Feria Muestras }\end{array}$ & $\begin{array}{c}\text { Playas } \\
\text { Veranes } \\
\text { Cimadevilla } \\
\text { Termas Romanas }\end{array}$ & \multicolumn{2}{|c|}{$\begin{array}{l}\text { Casino de Asturias } \\
\text { Elogio del Horizonte } \\
\text { Puerto Deportivo } \\
\text { Acuario-Poniente } \\
\text { Ruta del Cervigón } \\
\text { Parque Isabel la Católica }\end{array}$} \\
\hline
\end{tabular}

Fuente: Elaboración propia. 
FIGURA 4

ETAPAS DE APLICACIÓN DEL MODELO SMARTMAX

[1] Turistas con pernoctación en Gijón.

[2] Turistas con pernoctación en pequeños municipios colindantes con Gijón.

[3] Turistas con pernoctación en Gijón que no visitan atracciones externas.

Gijón

Destino

Inicial

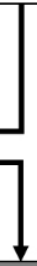

Gijón Destino Final

[4] Turistas con pernoctación en Gijón

que si visitan atracciones externas.

[5] Turistas con pernoctación en Gijón y otros destinos, antes y/o después.

[6] Turistas que no pernoctan en Gijón.

Fuente. Elaboración propia.

FIGURA 5

METODOLOGÍAS ESTADÍSTICAS DEL MODELO SMARTMAX

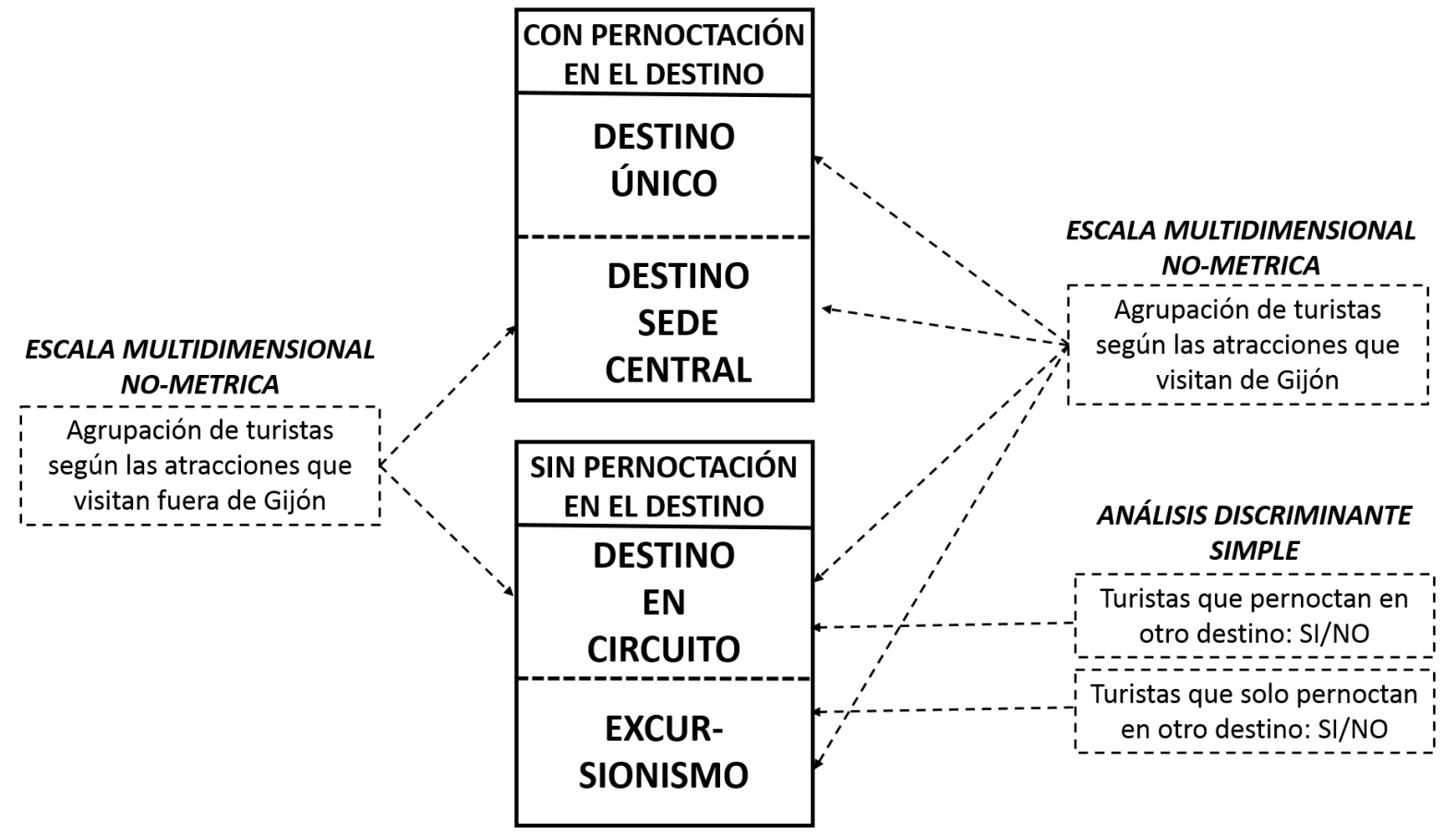

Fuente. Elaboración propia. 
diante el procedimiento CATPCA instalado en el IBM SPSS.

\section{RESUlTAdOS DEL ANÁLISIS CUANTITATIVO DEL PROTOCOLO SMARTMAX}

Previamente a exponer los resultados estadísticos de cada uno de los pasos del modelo propuesto, es conveniente indicar que:

1. En todos los Análisis Discriminantes Simples (ADS) se ha utilizado el método de pasos, que permite detectar las variables más discriminantes. Los dos ADS realizados han presentado valores de la lambda de Wilks elevados (siempre superiores al .978), con diferencias significativas al .01 en la Prueba de Box (ver tablas en el anexo).

2. Por su parte, los dos análisis del tipo Escala Multidimensional No Métrica (CAPTCA) realizados han presentado valores del alfa de Cronbach elevados (.902 y .964 respectivamente), así como porcentajes de la varianza acumulada suficientes: 72,157 para tres dimensiones en el primer caso; 71,159 para cinco dimensiones en el segundo (ver tablas en anexo).

Con los datos recepción de turistas de la región de Asturias, y considerando exclusivamente los casos de turistas alojados en una zona específica, y sin despla-
TABLA 3

CORRELACIONES ENTRE LOS TURISTAS DE LOS TRES PUNTOS CONSIDERADOS

\begin{tabular}{|c|c|c|}
\hline Se alojan & Visitan & Correlaciones \\
\hline Gijón & Cabo Peñas y Villaviciosa & $0,236^{* *}$ \\
\hline Cabo Peñas & Gijón y Villaviciosa & $0,216^{* *}$ \\
\hline Villaviciosa & Gijón y Cabo Peñas & $0,211^{* *}$ \\
\hline
\end{tabular}

$\left({ }^{* *}\right)$ Significativas al .000

Fuente: Elaboración propia.

zamiento a atracciones ajenas a misma (Destino Único), se han determinado tres áreas geo-turísticas principales: Gijón (44\%), Llanes $(17,4 \%)$ y Oviedo $(12,4 \%)$. Tras asumir la concepción de Gijón como destino turístico se ha procedido a estudiar la posible existencia "destinos satélites" del mismo: áreas geoturísticas más pequeñas, administrativamente independientes, generalmente colindantes, que se complementan y retroalimentan como un sistema turístico integrado.

Así, los resultados empíricos del modelo determinan que el destino Gijón opera con dos satélites: Villaviciosa y Cabo Peñas (Concejos de Gozón y Carreño). En ambos casos el número de turistas que visitan exclusivamente las atracciones de Gijón se aproxima al $64 \%$. Y, lo que es más importante, se han verificado correlaciones significativas entre los turistas de los tres puntos (ver tabla 3).

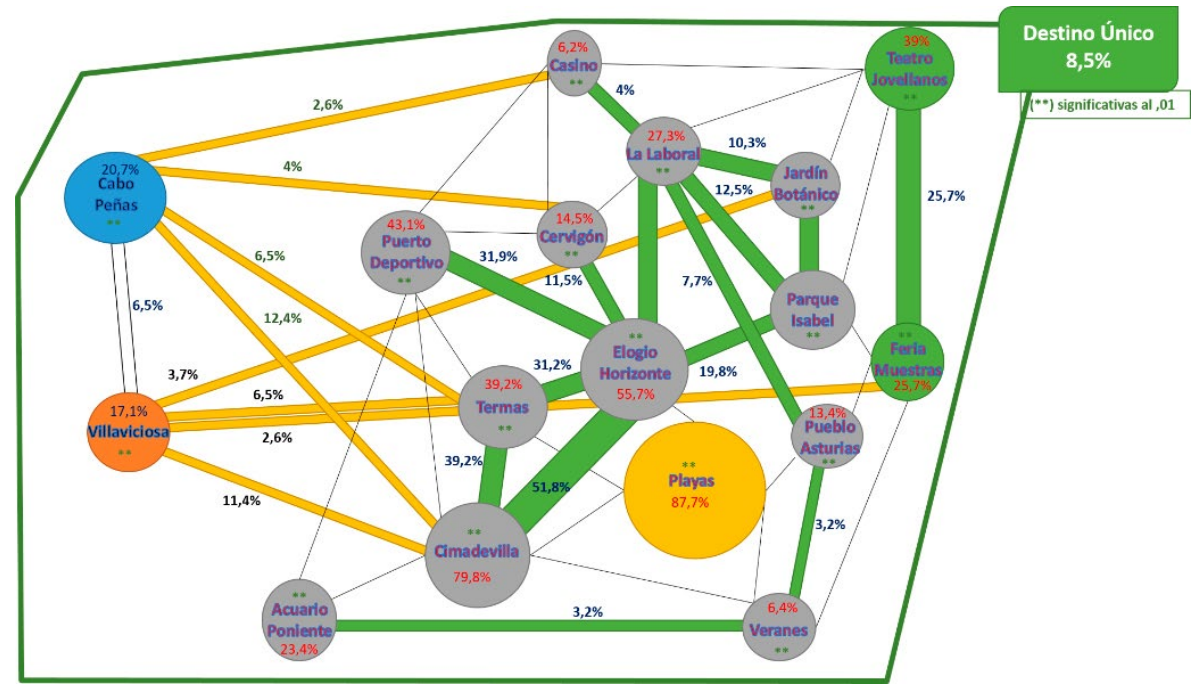

Fuente. Elaboración propia. 
Con tales resultados se amplía el área de influencia geográfica del destino inicial, para configurar un nuevo destino "Gijón Total", integrado por los municipios de Gijón, Villaviciosa y los dos de Cabo Peñas (Carreño y Luanco) (ver figura 6). Además, los resultados determinan el peso de la dimensión de Destino Único en el 8,5\% del total de los turistas, que utilizan cinco conjuntos de atracciones principales (ver figura 7).

El siguiente paso del protocolo considera el caso de los turistas que visitan atracciones externas al destino total Gijón. Así, los resultados del análisis CATPCA definen doce atracciones turísticas externas principales, que, además pueden ser agrupadas en cinco flujos principales:

- Flujo 1: que integra las visitas a Covadonga, Los Lagos y Cangas de Onís ("Covadonga”).

- Flujo 2: corresponde a las visitas a Lastres, Ribadesella y Llanes ("Villas marineras del oriente").

- Flujo 3: contiene las visitas hacia las villas marineras de Cudillero y de Luarca ("Villas marineras del occidente").
- Flujo 4: interrelaciona las visitas tanto a las localidades de los Picos de Europa como las rutas por sus montañas ("Picos de Europa”).

- Flujo 5: para las visitas a las otras dos grandes ciudades del centro de Asturias, Oviedo y Avilés ("Ciudades de Asturias").

La medición de la cuota de Gijón bajo la perspectiva de destino de sede central es del 29,2\% del total de visitantes. Que, además, puede ser desglosada entre los cinco flujos en cuestión (ver figura 8).

Los últimos análisis estadísticos del protocolo corresponden al caso de los turistas que pernoctan fuera del Destino Total Gijón: primero, compartiendo pernoctación con Gijón (Destino en Circuito); segundo, sin compartir (Destino de Excursionismo). El análisis discriminante simple primero (ADS1) determina la existencia de cuatro atracciones del destino Gijón visitadas por los turistas en circuito, en concreto (ver figura 9):

- El barrio pesquero de Cimadevilla, con alto peso, seguido del Elogio del Horizonte, Ruinas romanas de Veranes y la Ruta costera del Cervigón.

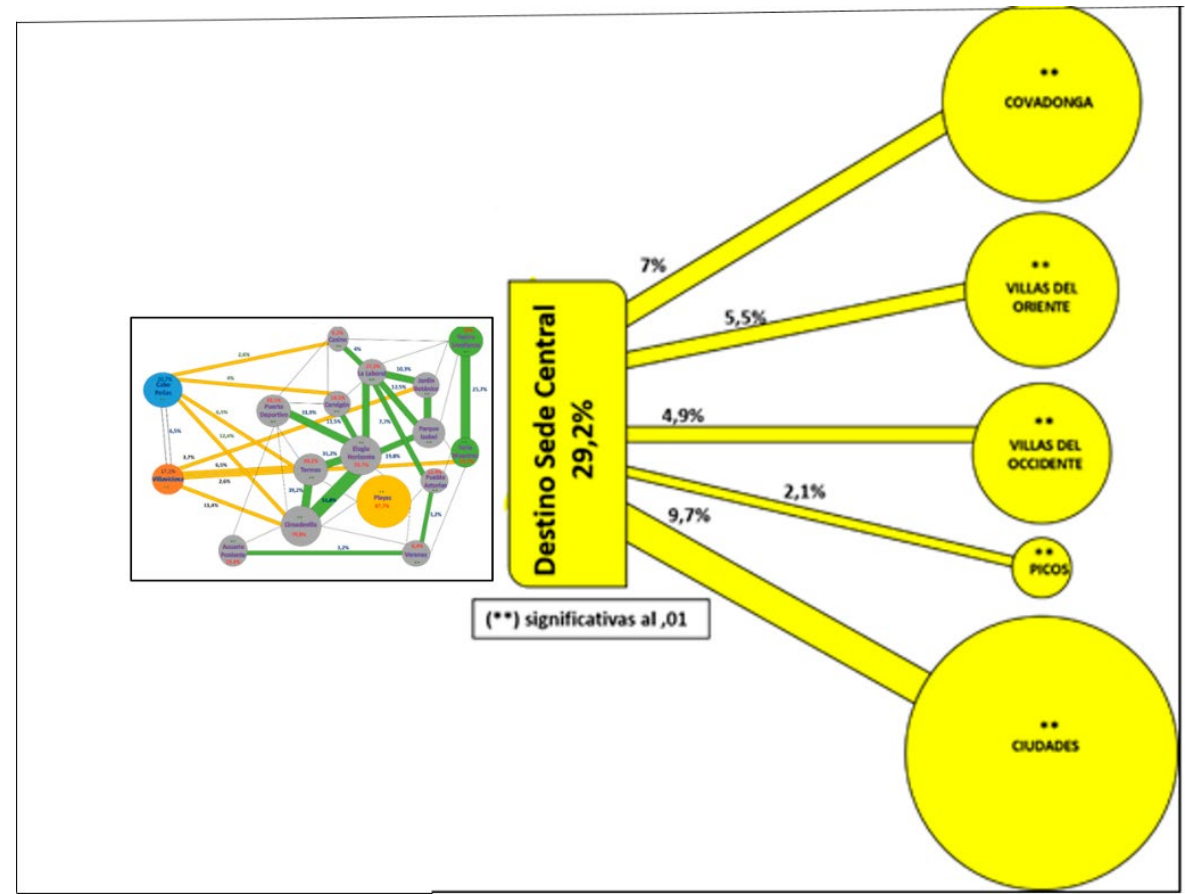


- Gijón como destino en circuito se relaciona de forma significativa y positiva con la región de Castilla-León y negativa con Cantabria (ambos casos al .5 de significación).

- El tamaño de Gijón como destino de circuito es del $5,9 \%$ sobre el total de visitantes. A su vez se distribuyen en: $86 \%$ del tipo sede central y $14 \%$ como destino único.

En relación al caso del Excursionismo debe destacarse como primer resultado su alto valor para Gijón: el $56,6 \%$ de sus visitantes son excursionistas. Por su parte, la aplicación de los ADS2 (orígenes de la pernoctación) y del ADS3 (atracciones de Gijón visitadas) indica que (ver Figura 10):

1. Provienen de cinco orígenes de pernoctación principales: Oviedo, Nava/Siero, Ribadesella, Castrillón y Avilés.

2. Visitan, de forma diferencial, cuatro principales atracciones turísticas: Teatro Jovellanos, Jardín Botánico, Playas y Cimadevilla.

En consecuencia, los resultados empíricos parecen avalar la mayoría de las hipótesis planteadas (ver tabla 4):

- H.1: Los tipos de flujos determinan la multi-dimensionalidad del destino turístico: Satélites, Destino Único, Sede Central, en Circuito y de Excursionismo.

FIGURA 9

SMARTMAX DE GIJÓN COMO DESTINO DE CIRCUITO

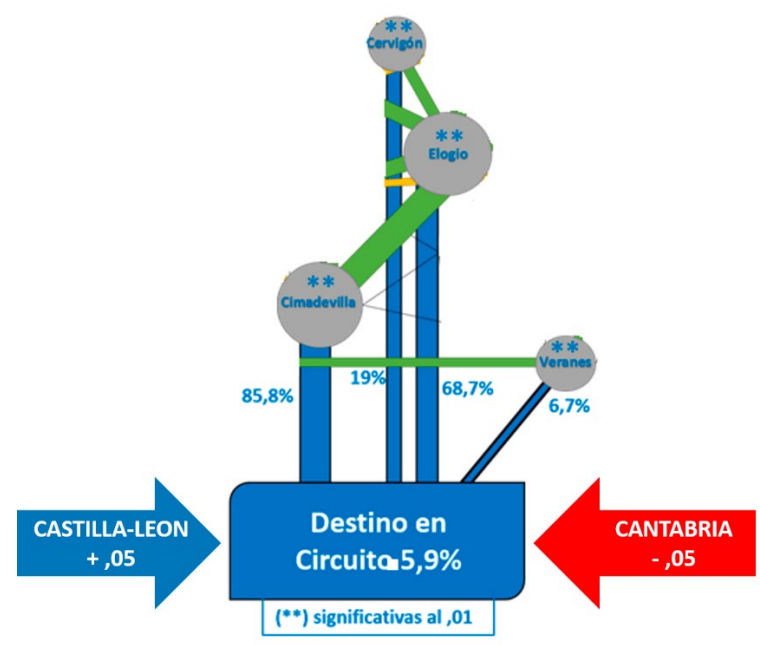

Fuente. Elaboración propia.
FIGURA 10

SMARTMAX DE GIJÓN COMO DESTINO DE EXCURSIONISMO

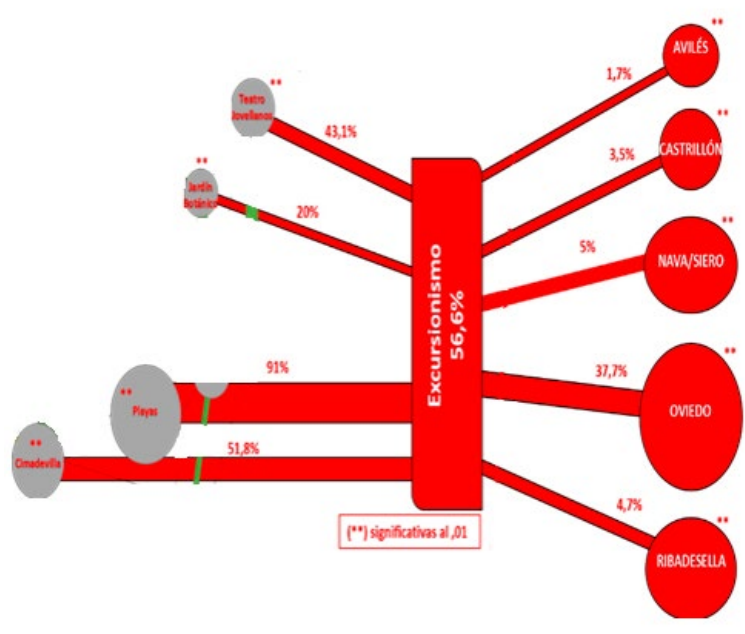

Fuente. Elaboración propia.

- H1.1: Los flujos internos y externos cortos hacia/ entre las atracciones amplían la dimensión geográfica del destino, como emisor (Sede Central) y como receptor (Excursionistas).

- H1.2: Una alta intensidad de flujos externos cortos hacia/entre las atracciones entre áreas colindantes amplían la dimensión geográfica del destino, mediante los sub-destinos satélites.

- H1.3: Los flujos externos largos hacia/entre los servicios turísticos amplían la dimensión geográfica del destino, como emisor y/0 como receptor (Destino en Circuito).

- H2: Las interrelaciones de los flujos internos y externos cortos hacia/entre las atracciones crean una cartera de experiencias turísticas principales del destino (en este caso, de 18 opciones).

\section{CONCLUSIONES}

Las concepciones clásicas del destino turístico han avanzado mucho en sus puntos de encuentro, pero, aun así, se sigue careciendo de una definición de consenso del destino turístico. Ni siquiera la OMT, al redactar sus definiciones, ha logrado una solución adecuada. Pese a ello las aportaciones académicas han sido numerosas en los últimos años. $Y$ en este contexto, el "Enfoque de Red" (Network) presenta un 
TABLA 4

CONTRASTES DE LAS HIPÓTESIS

\begin{tabular}{|c|c|c|c|}
\hline Hipótesis & Metodología & Significación & Resultados \\
\hline $\begin{array}{l}\text { H1: Existencia de } 5 \text { dimensiones del } \\
\text { destino. }\end{array}$ & $\begin{array}{l}\text { Matriz de Correlaciones } \\
\text { ADS1 } \\
\text { ADS2 }\end{array}$ & $\begin{array}{c}.000 \text { y } .000 \\
.000 \text { y } \lambda=0,961 \\
.000 \text { y } \lambda=0,976\end{array}$ & $\begin{array}{c}\text { Satélites: Villaviciosa, Carreño y } \\
\text { Luanco. } \\
\text { Destino Único }=8,5 \% \\
\text { Sede Central }=29,2 \% \\
\text { Circuito }=5,9 \% \\
\text { Excursión }=56,1 \%\end{array}$ \\
\hline H1.1: Movimientos entre Atracciones & $\begin{array}{l}\text { EMNM1 } \\
\text { EMNM2 }\end{array}$ & $\begin{array}{c}\alpha \text { cronbach }=.902 \\
\% \text { varianza }=72,16 \\
\alpha \text { cronbach }=.964 \\
\% \text { varianza }=71,15\end{array}$ & $\begin{array}{l}\text { Destino Único }=8,5 \% \\
\text { Sede Central }=29,2 \%\end{array}$ \\
\hline $\begin{array}{l}\text { H1.2: Ampliación de la zona } \\
\text { geoturística con otras de tipo Satélite }\end{array}$ & Matriz de Correlaciones & .000 y .000 & $\begin{array}{c}\text { Satélites: Villaviciosa, Carreño y } \\
\text { Luanco. }\end{array}$ \\
\hline $\begin{array}{l}\text { H1.3: Movimientos entre servicios de } \\
\text { pernocta }\end{array}$ & $\begin{array}{l}\text { ADS1 } \\
\text { ADS2 }\end{array}$ & $\begin{array}{l}.000 \text { у } \lambda=0,961 \\
.000 \text { у } \lambda=0,976\end{array}$ & $\begin{array}{c}\text { Pernocta } \\
\text { No Pernocta } \\
\text { Combina Pernoctación }\end{array}$ \\
\hline $\begin{array}{l}\text { H2: Tipologías de Experiencias } \\
\text { turísticas }\end{array}$ & EMNM1 EMNM2 & $\begin{array}{l}\alpha \text { cronbach }=.902 \\
\% \text { varianza }=72,16 \\
\alpha \text { cronbach }=.964 \\
\% \text { varianza }=71,15\end{array}$ & $\begin{array}{l}4 \text { en Destino único } \\
5 \text { en Sede Central } \\
4 \text { en Circuito } \\
5 \text { en Excursionismo }\end{array}$ \\
\hline
\end{tabular}

Fuente: Elaboración propia.

aceptable nivel de consenso, porque es capaz de integrar, a nivel intra/inter, las relaciones entre los distintos tipos de agentes, atracciones, servicios, imagen e, incluso, estrategias turísticas.

Asimismo, la aceptación del factor "experiencia", como fundamento del concepto de turismo, ha favorecido una mejor comprensión del porqué y del cómo los turistas eligen y utilizan las atracciones y los servicios turísticos. Además, la eclosión de las tecnologías "mobile", que facilitan las conexiones "online y ontime", plantea la existencia de una "experiencia de segunda generación", que integra el fenómeno de la "co-creación" como parte del concepto de destino turístico. En este sentido, la co-creación de las experiencias turísticas implica aceptar un paradigma de variabilidad y multi-dimensionalidad del destino turístico: el turista busca una experiencia auténticamente única y personal, construye "su" concepto de destino, totalmente distinto al de cualquier otro turista, e incluso al suyo en cualquier otro momento.

El conocido juego magnético SmartMax es, en realidad, un montaje en red. Sencillo y didáctico de comprender para los gestores y profesionales turísticos. Pero, además, dotado de ciertas capacidades estadísticas, a pesar de las limitaciones métricas que tienen los destinos, problemas de medida muy habituales al intentar aplicar los procedimientos de Análisis de Red (SNA).
El juego SmartMax opera con los componentes principales de un destino turístico, a saber:

(a) Los nodos, representativos de las atracciones y/o de los servicios turísticos. En sus diversas acepciones -tamaño, tipología experiencial-, son resueltas por nodos de diferentes tamaños y colores.

(b) Los conectores, identificativos de los movimientos inherentes al destino, sea desde el origen de residencia del turista, desde otro destino, desde un nodo de servicios y/o un nodo de atracciones turísticas. También, sus diversas posibilidades -largura, anchura y tipología- son representadas por piezas conectoras de distinto tamaño y color.

(c) Incorpora la gran importancia de la imagen percibida del destino por el turista. Que será la "plantilla mental" que el turista siga para elegir, y montar, los nodos y los conectores.

La aplicación empírica del SmartMax al caso de Gijón ha demostrado su practicidad. El uso de los modelos estadísticos no-paramétricos, como la Escala Multidimensional No-métrica y el Análisis Discriminante Simple dotan al protocolo de cierto rigor estadístico.

SmartMax propone un protocolo de estudio práctico del destino mucho más allá de las macro-esta- 
dísticas tradicionales -tasa de ocupación, pernoctaciones, transporte, paquetes ...- facilitando un análisis a nivel micro del turista y del destino. Algo que la metodología "Network Analysis" no ha sido capaz de desarrollar de una manera sencilla de comprender y de realizar.

Su aplicación práctica es perfectamente extrapolable y realizable para cualquier destino turístico. Los autores, de hecho, han realizado una versión del juego para el caso de Cangas de Onís (España), y se encuentran, actualmente replicando el protocolo para el caso de Oviedo. La única limitación es disponer de una base de datos suficiente sobre el uso de las atracciones y de los servicios turísticos por parte de los visitantes al destino. Tal base de datos puede proceder de encuestas en destino, como es el caso, pero también de otros orígenes más objetivos como las tarjetas turísticas, las apps turísticas o las importantes aplicaciones de la geolocalización.

En tanto que sus utilidades pueden amplias para los distintos agentes turísticos. Para los investigadores académicos como metodología práctica alternativa a las limitaciones métricas del SNA. Además de como línea abierta de trabajo para profundizar en el desarrollo de más componentes y, sobre todo, de indicadores de comprensión del destino, como densidad, geometría, amplitud, densidad, diversidad. Para las entidades estadísticas puede servir de guía para la

\section{Biblografía}

Alberca, F.A. (2016). Una mirada al destino turístico y sus aspectos materiales e inmateriales. Cultura (30), 65-85.

Baggio, R., y Sainaghi, R. (2014). Analyzing tourist flows by mapping time series into networks. Tourists as Consumers, Visitors, Travellers, 1-4.

Belghiti, S., Ochs, A., Lemoine, J. F., y Badot, O. (2017). The Phygital Shopping Experience: An Attempt at Conceptualization and Empirical Investigation. En Academy of Marketing Science World Marketing Congress (61-74). Nueva York, USA: Ed. Springer.

Beritelli, P. y Bieger, T. (2014). From destination governance to destination leadership - defining and exploring the significance with the help of a systemic perspective. Tourism Review, 69 (1), 25-46. obtención de datos más adaptados a la comprensión práctica de los destinos.

Aunque, sin duda, la principal utilidad sería para los planificadores y gerentes de los destinos turísticos, que dispondrían de un mejor conocimiento de los distintos niveles del destino, de los movimientos y modelos de co-creación de los turistas con los recursos, de un generador de ideas para desarrollar productos turísticos adaptados a las rutas de los turistas, para comprender cierta dinámica de transformación del destino en el tiempo, y las diferencias de forma según temporadas. Desde los niveles más altos de la administración turística puede servir para poner en marcha mecanismos de cooperación entre zonas geoturísticas y otros destinos. A los empresarios les permitiría un mejor conocimiento de los comportamientos de sus clientes y para desarrollar clústeres de colaboración coherentes a los flujos de los turistas. $\mathrm{Y}$, en todos, estos casos, para generar nuevas acciones de imagen de los destinos basados en estrategias de competencia entre instituciones autonómicas, patronatos, fundaciones y similares.

Finalmente debe considerarse su aplicación a otras posibles actividades de servicios con dispersión territorial, como puede ser el caso de las zonas hosteleras, o de los centros y parques turísticos, toda vez que existen nodos y flujos concretos en los mismos.

Bonetti, E., Petrillo, C. S., y Simoni, M. (2006). Tourism system dynamics: A multi-level destination approach. En Tourism local systems and networking (111-131). Stirling, UK: Elsevier.

Boullón, R. C. (2004). Planificación del Espacio Turístico. Barcelona, España: Ed. Trillas.

Buhalis, D. (2003). eTourism: Information technology for strategic tourism management. Londres, UK: Pearson Education.

Buonincontri, P.; Morvillo, A.; Okumus, F. y Niekerk, M. van (2017). Managing the experience co-creation process in tourism destinations: Empirical findings from Naples. Tourism Management (62), 264-277.

Casanueva, C., Gallego, A. y García-Sánchez, M.R. (2014). Social Network Analysis in Tourism. Current Issues in Tourism, 19 (12), 469-484. 
Chathoth, P.K., Ungson, G.R.; Harrington, R.J. y Chan, E.S.W. (2016). Co-creation and higher order customer engagement in hospitality and tourism services: A critical review. International Journal of Comtemporary Hospital Management, 28(2), 222-245.

Cooper, C., Fletcher, J., Wanhill, S., Gilbert, D. y Shepherd, R. (2005). Tourism: Principles and Practice. Londres, UK: Pearson Education.

D`Agata, R., Gozzo, S. y Tomaselli, V. (2013). Network analysis approach to map tourism mobility. Qual Quant (47), 2167-3184.

Denicolai, S., Cioccarelli, G., y Zucchella, A. (2010). Resource-based local development and networked core-competencies for tourism excellence. Tourism Management, 31 (2), 260-266.

Framke, W. (2002). The Destination as a Concept: A Discussion of the Business-related Perspective versus the Socio-cultural Approach in Tourism Theory. Scandinavian Journal of Hospitality and Tourism, 2 (2), 92-108.

Füller, J. (2010). Refining virtual co-creation from a consumer perspective. California Management Review, 52 (2), 98-122.

García, M.A. (2018). La co-creación a través de los social-media: Efecto en la imagen de Cuenca como destino turístico. Tesis Doctoral, Universidad de Castilla-La Mancha, Albacete.

González, L. (28, 05, 2017). Cinco características que diferencian al turista 3.0. Recuperado en: http:// www.cognodata.com/notas_de_prensa

Gretzel, U., Werthner, H., Koo, C., y Lamsfus, C. (2015). Conceptual foundations for understanding smart tourism ecosystems. Computers in Human Behavior (50), 558-563.

Gretzel, U., Zhong, L., y Koo, C. (2016). Application of smart tourism to cities. International Journal of Tourism Cities, 1(3), 216-233.

Grönroos, C. (2008). Service logic revisited: who creates value? And who co-creates? European Business Review, 20 (4), 298-314.

Ind, N., Iglesias, O., y Schultz, M. (2013). Building brands together: Emergence and outcomes of co-creation. California Management Review, 55 (3), 5-26.

Kambil, A., Friesen, G., y Sundaram, A. (1999). Cocreation: A new source of value. Outlook Magazine, 3 (2), 23-29.
Keum, K. (2010). Tourism flows and trade theory: A panel data analysis with the gravitiy model. The Annals of Regional Science (44), 541-557.

Kladou, S., y Kehagias, J. (2014). Assessing destination brand equity: An integrated approach. Journal of Destination Marketing \& Management, 3 (1), 2-10.

Kotler, P., Kartajaya, H., y Setiawan, I. (Eds.). (2017). Marketing 4.0: Moving from Traditional to Digital. Hoboken, USA: John Wiley y Sons, Inc.

Li, Y.; Hu C.; Huang, C. y Duan, L. (2017), The concept of smart tourism in the context of tourism information services, Tourism Management (58), 293-300.

Liu, W., Guillet, B. D., Xiao, Q. y Law, R. (2014). Globalization or localization of consumer preferences: The case of hotel room booking. Tourism Management (41), 148-157.

Lohmann, G. y Pearce, D.G. (2010). Conceptualizing and operationalizing nodal tourism functions. Journal of Transport Geography (18), 266-275.

Manente, M. y Cerato, M. (2000). Understanding the Destination as a system. En From Destination to Destination Marketing and Management. Designing and Repositioning Tourism Products (131146). Venecia, Italia: CISET.

Marrocu, E. y Paci, R. (2013). Different tourists to different destinations. Evidence from spatial interaction models. Tourism Management (30), 71-83

Martínez, B. y Rojo, R. (2013). Destinos turísticos. Guía, información y asistencias turísticas. Agencias de viajes y gestión de eventos. Madrid, España: Paraninfo.

Martini, A., Massa, S., y Testa, S. (2014). Customer co-creation projects and social media: The case of Barilla of Italy. Business Horizons, 57 (3), 425-434.

Merilainen, K., y Lemmetyinen, A. (2011). Destination network management: a conceptual analysis. Tourism Review, 66 (3) 25-3.

Merinero, R., y Pulido, J. (2009). Desarrollo turístico y dinámica relacional. Madrid, España: Ed. Metodología.

Moragues, D. (2006): Turismo, Cultura y Desarrollo. Madrid, España: Ed. OEl. 
Morley C., Rosselló, J. y Santana, M. (2014). Gravity models fortourism demand: Theory and use. Annals of Tourism Research (48), 1-10.

Navarro, D. (2015). Recursos turísticos y atractivos turísticos: Conceptualización y valoración. Cuadernos de Turismo (35), 335-357.

OMT (Ed.) (2008). Entender el Turismo: Glosario Básico. Madrid, España.

Pearce, D.G. (2012). Frameworks for Tourism Research. Wallingford, UK: CABI.

Pearce, D.G. (2014). Toward an Integrative Conceptual Framework of Destinations. Journal of Travel Research (53), 141-153.

Rabari, C. y Storper, M. (2015). The digital skin of cities: Urban theory and research in the age of the sensored and metered city, ubiquitous computing and big data. Cambridge Journal of Regions, Economy and Society, 8 (1), 27-42.

Richards, G. (2011). Creativity and tourism: The state of the art. Annals of Tourism Research, 38(4), 1225-1253.

Ritzer, G., Dean, P., y Jurgenson, N. (2012). The coming of age of the prosumer. American Behavioral Scientist, 56(4), 379-398.

Sang-Hyun, L., Jin- Yong, C., Seung-Hwan, Y. e YunGyeong, O. (2013). Evaluating spatial centrality for integrated tourism management in rural areas using GIS and network analysis. Tourism Management (34), 14-24.

Saraniemi, S. y Kylanen, M. (2011). Problematizing the concept of Tourism Destination. Journal of Travel Research, 50 (2), 133-143.

Sorensen, F., y Jensen, J. (2015). Value creation and knowledge development in tourism experience encounters. Tourism Management (46), 336-346.

Vargo, S., y Lusch, R. (2008). Service-dominant logic: continuing the evolution. Journal of the Academy of Marketing Science, 36 (1), 1-10.

Vera, F. y Baños, C. (1997). Renovación y reestructuración de los destinos turísticos consolidados del litoral: Las prácticas recreativas en la evolución del espacio turístico. Boletín de la Asociación de Geógrafos Españoles (53), 329-353.

Vera, J.F., López, F., Marchena, M.J. y Antón, S. (2011). Análisis Territorial del Turismo y Planificación de Destinos Turísticos. Valencia, España: Ed. Tirant Humanidades.

Violier, P. (2009). Proposition pour un modèle d'analyse dynamique des lieux touristiques. Destinations et Territoires (1), 26-37.

Yang, W.K.F. (2012). A spatial Econometric Approach to Model Spillover Effects in Tourism Flows. Journal of Travel Research (51), 768-778. 